\title{
Self-Assembly of Surfactant Vesicles that Transform into Viscoelastic Wormlike Micelles upon Heating
}

\author{
Tanner S. Davies, Aimee M. Ketner, and Srinivasa R. Raghavan* \\ Contribution from the Department of Chemical \& Biomolecular Engineering, \\ University of Maryland, College Park, Maryland 20742-2111
}

Received January 2, 2006; E-mail: sraghava@eng.umd.edu

\begin{abstract}
Unilamellar vesicles are observed to form in aqueous solutions of the cationic surfactant, cetyl trimethylammonium bromide (CTAB), when 5-methyl salicylic acid $(5 \mathrm{mS})$ is added at slightly larger than equimolar concentrations. When these vesicles are heated above a critical temperature, they transform into long, flexible wormlike micelles. In this process, the solutions switch from low-viscosity, Newtonian fluids to viscoelastic, shear-thinning fluids having much larger zero-shear viscosities (e.g., 1000-fold higher). The onset temperature for this transition increases with the concentration of $5 \mathrm{mS}$ at a fixed CTAB content. Small-angle neutron scattering (SANS) measurements show that the phase transition from vesicles to micelles is a continuous one, with the vesicles and micelles coexisting over a narrow range of temperatures. The tunable vesicle-to-micelle transition and the concomitant viscosity increase upon heating may have utility in a range of areas, including microfluidics, controlled release, and tertiary oil recovery.
\end{abstract}

\section{Introduction}

Vesicles and micelles represent two of the important classes of self-assembled structures that can be formed by amphiphiles in dilute or semidilute solution. ${ }^{1}$ Vesicles are hollow spheres enclosed by a bilayer of the amphiphiles and are commonly used to encapsulate labile hydrophilic molecules within their interior. Micelles tend to occur in a range of morphologies, including spherical, ellipsoidal, and cylindrical structures. Cylindrical micelles that are very long and flexible are referred to as "wormlike micelles", and their formation is linked to the emergence of viscoelasticity in the solution. ${ }^{2,3}$ Due to their viscoelastic properties, wormlike micelles have found applications in many areas, such as in personal care products and in the oilfield industry. ${ }^{4}$ Frequently, they are used in moderate to high-temperature environments.

In this paper, we report a thermoreversible transition from unilamellar vesicles to wormlike micelles with increasing temperature in a surfactant fluid. This transition results in a dramatic (1000-fold) increase in the fluid viscosity. The system described here is a simple one, involving the well-known cationic surfactant, cetyl trimethylammonium bromide (CTAB), and a relatively common organic derivative, 5-methyl salicylic acid $(5 \mathrm{mS})$. Fluids that viscosify or gel upon heating are of great interest for biomedical and drug delivery applications, ${ }^{5}$ for flow control and separations using microfluidic devices, ${ }^{6}$ and as

(1) Evans, D. F.; Wennerstrom, H. The Colloidal Domain; Wiley-VCH: New York, 2001

(2) Cates, M. E.; Candau, S. J. J. Phys.: Condens. Matter 1990, 2, 68696892.

(3) Hoffmann, H. In Structure and Flow in Surfactant Solutions; Herb, C. A., Prud'homme, R. K., Eds.; American Chemical Society: Washington, DC, 1994; pp 2-31.

(4) Yang, J. Curr. Opin. Colloid Interface Sci. 2002, 7, 276-281.

(5) Jeong, B.; Lee, K. M.; Gutowska, A.; An, Y. H. H. Biomacromolecules 2002, 3, 865-868.

10.1021/ja060021e CCC: $\$ 33.50$ @ 2006 American Chemical Society hydraulic fracturing fluids in enhanced oil recovery. ${ }^{7}$ The simplicity, low cost, and ease of preparation of the present system might make it attractive for some of these applications.

Mixtures of CTAB and $5 \mathrm{mS}$ have been previously studied by Davis et al. ${ }^{8}$ The focus of their study was on the structural transition from spherical to wormlike micelles as the $5 \mathrm{mS}$ :CTAB molar ratio was increased from zero to beyond equimolar. Surprisingly, at a molar ratio around 1.1, the authors found instead that the solution contained unilamellar vesicles. This result was inferred from a cryo-transmission electron microscopy (cryo-TEM) image, which showed vesicles around $100 \mathrm{~nm}$ in diameter. Detailed investigations of vesicle formation were not reported in this study. In a subsequent paper, Davis, Zakin, and co-workers ${ }^{9}$ again used cryo-TEM to infer the presence of vesicles, this time in mixtures of CTAB and the sodium salt of 3-methyl salicylic acid $(3 \mathrm{mS})$. These vesicles were reported to transform into cylindrical micelles upon shearing.

Vesicle-to-wormlike micelle transitions with increasing temperature have been described previously in only a couple of systems to our knowledge. ${ }^{10,11}$ In the one studied by Manohar, Candau, and co-workers, ${ }^{10}$ the surfactant was obtained by mixing equimolar amounts of CTAB with sodium 3-hydroxynaphthalene 2-carboxylate (SHNC), followed by removal of excess counterions. This surfactant when added to water at room

(6) Kan, C. W.; Doherty, E. A. S.; Barron, A. E. Electrophoresis 2003, 24, 4161-4169.

(7) Dantas, T. N. C.; Santanna, V. C.; Neto, A. A. D.; Neto, E. L. B.; Moura M. Colloid Surf. A 2003, 225, 129-135.

(8) Lin, Z.; Cai, J. J.; Scriven, L. E.; Davis, H. T. J. Phys. Chem. 1994, 98 5984-5993.

(9) Zheng, Y.; Lin, Z.; Zakin, J. L.; Talmon, Y.; Davis, H. T.; Scriven, L. E. J. Phys. Chem. B 2000, 104, 5263-5271.

(10) Hassan, P. A.; Valaulikar, B. S.; Manohar, C.; Kern, F.; Bourdieu, L.; Candau, S. J. Langmuir 1996, 12, 4350-4357.

(11) Buwalda, R. T.; Stuart, M. C. A.; Engberts, J. Langmuir 2000, 16, 67806786. 
temperature assembled into multilamellar vesicles (MLVs) around $1-10 \mu \mathrm{m}$ in diameter. Due to the formation of these large MLVs, the solutions were highly turbid and quite viscous (viscosities ca. 100 times that of water). Upon increasing the temperature, the samples transformed into clear solutions containing wormlike micelles, thereby leading to an increase in viscosity by about an order of magnitude. It is not clear if the MLVs in these samples at low temperatures were stable or if they aggregated and eventually phase-separated to form a lamellar phase.

The present study with the $\mathrm{CTAB} / 5 \mathrm{mS}$ system began in an attempt to reproduce the result of Davis et al. ${ }^{8}$ in terms of forming unilamellar vesicles. Subsequently, we detected by visual observation a dramatic increase in viscosity when some of these samples were heated. This prompted us to carry out a systematic investigation of $\mathrm{CTAB} / 5 \mathrm{mS}$ mixtures over a range of compositions and temperatures. In our studies, we employ a combination of visual observations, turbidimetry, rheological techniques, and small-angle neutron scattering (SANS) measurements. Our results unambiguously demonstrate the formation of vesicles in these solutions at room temperature, and furthermore, we show that the vesicles undergo a continuous phase transition to wormlike micelles with increasing temperature. Finally, we propose a tentative mechanism based on an adsorption-desorption equilibrium to explain these results.

\section{Results}

CTAB/5mS Solutions: Studies at Room Temperature. We begin by discussing the phase behavior of CTAB $/ 5 \mathrm{mS}$ mixtures at a fixed [CTAB] of $12.5 \mathrm{mM}$. Figure 1 shows two types of data, the zero-shear viscosity $\eta_{0}$ and the optical density, as a function of $[5 \mathrm{mS}]$. The optical density was recorded at a wavelength of $500 \mathrm{~nm}$, where CTAB and $5 \mathrm{mS}$ absorb minimally, so that any changes in this quantity are due to the scattering of light by nanostructures in solution. Consider first the results for [5mS] below ca. $15 \mathrm{mM}$. At very low [5mS] $(<5 \mathrm{mM})$, the samples are clear solutions with a low optical density and a low viscosity close to that of water $(1 \mathrm{mPa} \cdot \mathrm{s})$. As [5mS] is increased, the viscosity grows by 5 orders of magnitude. The resulting samples are clear, viscoelastic solutions and show an ability to trap bubbles for long periods of time (see photograph of a typical sample in Figure 1). These samples also exhibit flow-birefringence, that is, they show streaks of light under crossed polarizers upon shaking. Both the viscoelasticity and the flow-birefringence are characteristics of wormlike micelles. ${ }^{3}$ The results imply that $5 \mathrm{mS}$ induces the growth of micelles, which is precisely as expected for these salicylic acid derivatives. ${ }^{8,12}$ With further increase in $[5 \mathrm{mS}]$, the viscosity in Figure 1 reaches a peak and thereafter drops precipitously. Similar viscosity peaks are ubiquitous in the wormlike micelle literature and are believed to signify a transition from linear to branched wormlike micelles, although this is still a matter of debate. ${ }^{3}$

The unusual results in our study are obtained for $[5 \mathrm{mS}]$ above $15 \mathrm{mM}$. At these concentrations, the samples show a bluish hue, and their viscosity drops to that of water (see photograph of a typical sample in Figure 1; note that it is a clear and homogeneous solution). The bluish color is a manifestation of the Tyndall effect due to the presence of large scatterers in

(12) Shikata, T.; Hirata, H.; Kotaka, T. Langmuir 1989, 5, 398-405.
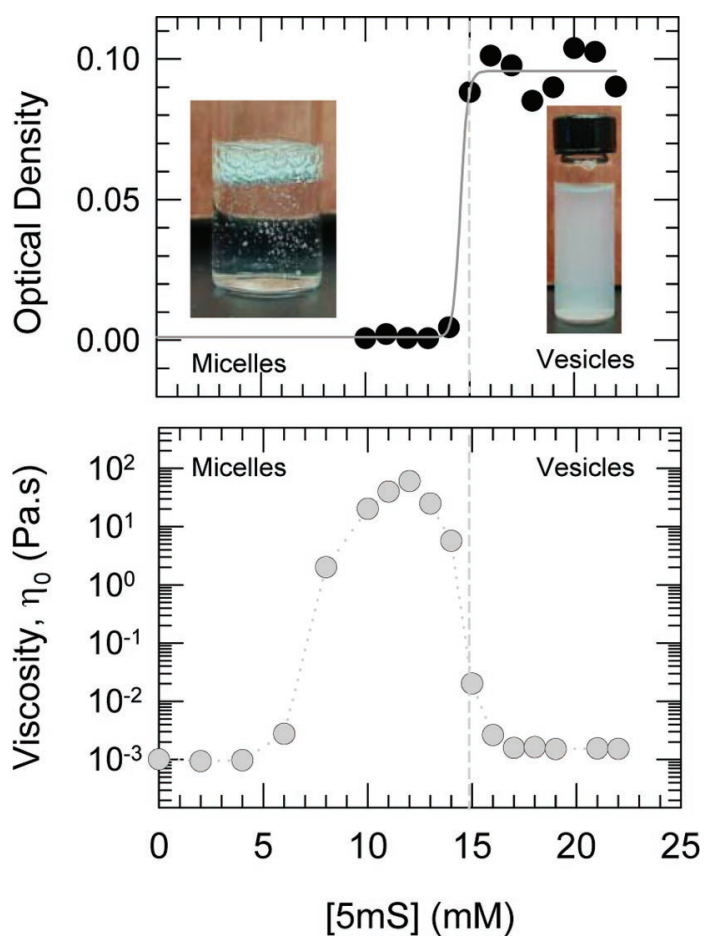

Figure 1. Phase behavior at $25^{\circ} \mathrm{C}$ of $\mathrm{CTAB} / 5 \mathrm{mS}$ mixtures at a fixed $\mathrm{CTAB}$ concentration of $12.5 \mathrm{mM}$ and varying concentrations of $5 \mathrm{mS}$. The top plot shows the optical density, which quantifies the amount of light scattered from the sample. The bottom plot shows the zero-shear viscosity obtained from steady-shear rheology. Samples containing around $10 \mathrm{mM} 5 \mathrm{mS}$ are colorless and highly viscous and show a tendency to trap bubbles, as seen from the photograph on the left. In contrast, samples with greater than ca. $15 \mathrm{mM} 5 \mathrm{mS}$ have a low viscosity akin to water and appear bluish due to light scattering, as seen from the photograph on the right.

solution, and it is generally seen for solutions containing vesicles. ${ }^{13}$ The onset of the bluish color is indicated by the sharp rise in optical density in Figure 1. At higher [5mS], the optical density and the viscosity both reach plateaus. Taken together, the data provide preliminary evidence for a phase transition from micellar structures to vesicles with increasing $[5 \mathrm{mS}]$. Note that Figure 1 extends only up to ca. $23 \mathrm{mM}$ [5mS], which represents a solubility limit for $5 \mathrm{mS}$ in water at room temperature in the presence of $12.5 \mathrm{mM}$ CTAB. Indeed, organic acids, such as $5 \mathrm{mS}$, are sparingly soluble in water at neutral $\mathrm{pH}$. In the absence of surfactant, we measured the aqueous solubility of $5 \mathrm{mS}$ at 25 ${ }^{\circ} \mathrm{C}$ to be $7.4 \mathrm{mM}$.

To further elucidate the microstructures in these samples, we resorted to SANS. CTAB/5mS samples were prepared in $\mathrm{D}_{2} \mathrm{O}$ to achieve the needed contrast between scatterers and solvent. Samples in $\mathrm{D}_{2} \mathrm{O}$ were found to be visually and rheologically identical to those prepared with $\mathrm{H}_{2} \mathrm{O}$. Figure 2 shows SANS spectra $(I$ vs $q$ ) for $12.5 \mathrm{mM} \mathrm{CTAB}$ samples containing different amounts of $5 \mathrm{mS}$. The data for low $[5 \mathrm{mS}](5$ and $10 \mathrm{mM})$ asymptote to a plateau at low $q$ and essentially correspond to micelles. In contrast, there is no plateau at the higher $[5 \mathrm{mS}]$, with the 15 and $20 \mathrm{mM}$ samples showing a $q^{-2}$ decay of the intensity at low $q$. Such a decay is a signature of scattering from vesicle bilayers. ${ }^{14}$ Importantly, the $q^{-2}$ decay occurs only for samples within the vesicle region in Figure 1 (i.e., the region

(13) Kaler, E. W.; Murthy, A. K.; Rodriguez, B. E.; Zasadzinski, J. A. N. Science 1989, 245, 1371-1374.

(14) Hubbard, F. P.; Santonicola, G.; Kaler, E. W.; Abbott, N. L. Langmuir 2005, 21, 6131-6136. 


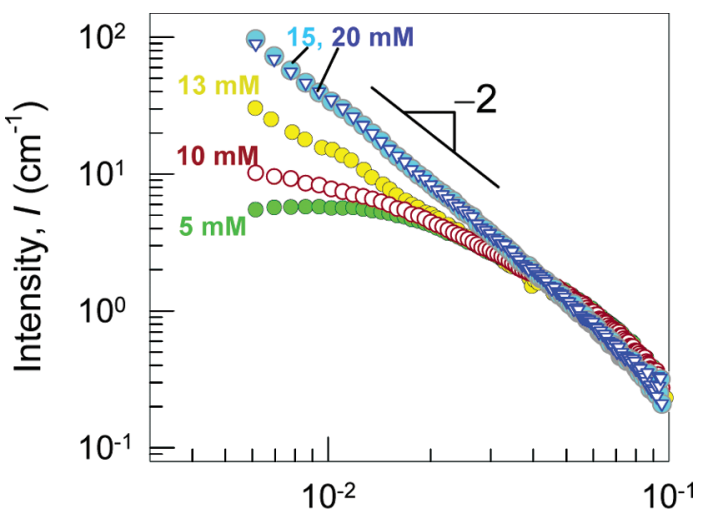

Wave vector, $q\left(\AA^{-1}\right)$

Figure 2. SANS scattering at $25{ }^{\circ} \mathrm{C}$ from $\mathrm{CTAB} / 5 \mathrm{mS}$ mixtures at a fixed $\mathrm{CTAB}$ concentration of $12.5 \mathrm{mM}$ and varying concentrations of $5 \mathrm{mS}$.

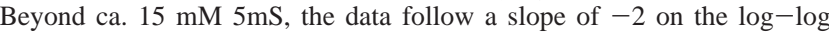
plot, which is indicative of scattering from vesicles.

of bluish, nonviscous samples). Thus, the SANS data support our hypothesis of a phase transition from micelles to vesicles with increasing $[5 \mathrm{mS}]$. Further analysis of the SANS data, including quantification of vesicle sizes, is described in the Discussion section.

CTAB/5mS Solutions: Studies as a Function of Temperature. We have indicated that certain $\mathrm{CTAB} / 5 \mathrm{mS}$ mixtures contain vesicles at room temperature. Next, we describe the unusual behavior exhibited by these solutions upon heating. The solutions are visually observed to transform from bluish and nonviscous at low temperatures to colorless, perceptibly viscous and flow-birefringent at high temperatures. Systematic studies as a function of temperature for a sample containing $12.5 \mathrm{mM}$ $\mathrm{CTAB}$ and $20 \mathrm{mM} 5 \mathrm{mS}$ are reported in Figure 3. Here, again, both the optical density at $500 \mathrm{~nm}$ and the zero-shear viscosity $\eta_{0}$ are shown, this time as a function of temperature. Note from Figure 1 that this sample falls in the vesicle region of the phase diagram at $25^{\circ} \mathrm{C}$, and at low temperatures (between 25 and ca. $48^{\circ} \mathrm{C}$ ), the sample remains bluish and scatters light strongly. Around $48{ }^{\circ} \mathrm{C}$, the bluish hue slowly begins to fade, and the sample transforms to a clear, colorless solution. In turn, the optical density drops sharply and falls to nearly zero by about $54{ }^{\circ} \mathrm{C}$. Corresponding changes also occur in the rheology-as the sample clears, it also becomes more viscous, and the zeroshear viscosity $\eta_{0}$ increases by a factor of about 500 . At higher temperatures, the sample continues to remain clear and viscous, while the viscosity drops with temperature. The data presented here suggest a transition from vesicles to wormlike micelles upon heating, and we will presently use rheology and SANS to study and confirm such a transition.

The rheological response of the above CTAB $/ 5 \mathrm{mS}$ sample at various temperatures is shown in Figure 4. At low temperatures, the sample exhibits Newtonian behavior; that is, its viscosity is independent of shear rate, and the value of this viscosity is close to that of water (Figure 4a). This response is consistent with the presence of discrete unilamellar vesicles, and the same response persists from 25 to ca. $50{ }^{\circ} \mathrm{C}$. At ca. 55 ${ }^{\circ} \mathrm{C}$, however, the sample switches to a shear-thinning response, with a plateau in the viscosity at low shear rates, followed by a drop in viscosity at higher shear rates. Note also that the zeroshear viscosity is orders of magnitude higher at this temperature.

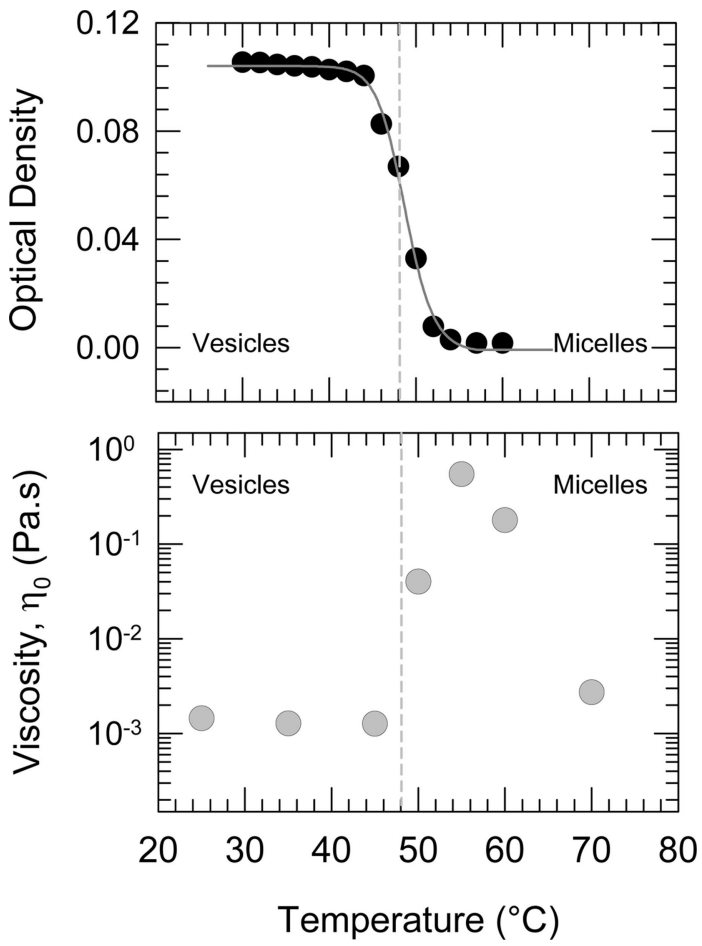

Figure 3. Phase behavior versus temperature of a sample containing 12.5 mM CTAB and $20 \mathrm{mM} \mathrm{5mS.} \mathrm{The} \mathrm{optical} \mathrm{density} \mathrm{(top)} \mathrm{and} \mathrm{the} \mathrm{zero-shear}$ viscosity (bottom) are shown. The sharp increase in viscosity and the corresponding decrease in optical density suggest a vesicle-to-micelle transition with increasing temperature.
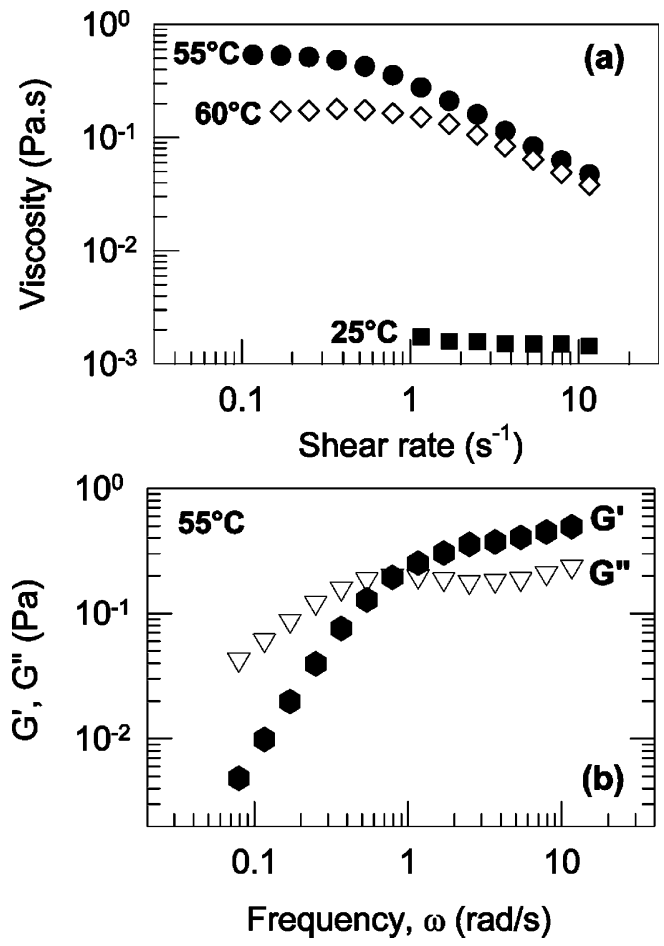

Figure 4. Rheology of a $12.5 \mathrm{mM} \mathrm{CTAB}+20 \mathrm{mM} 5 \mathrm{mS}$ sample at various temperatures. The steady-shear rheological response at three different temperatures is shown in (a), and the dynamic rheological response at 55 ${ }^{\circ} \mathrm{C}$ is shown in (b).

These rheological changes are consistent with a transition from vesicles to wormlike micelles. The corresponding dynamic rheological response at $55^{\circ} \mathrm{C}$ is presented in Figure $4 \mathrm{~b}$ as plots 


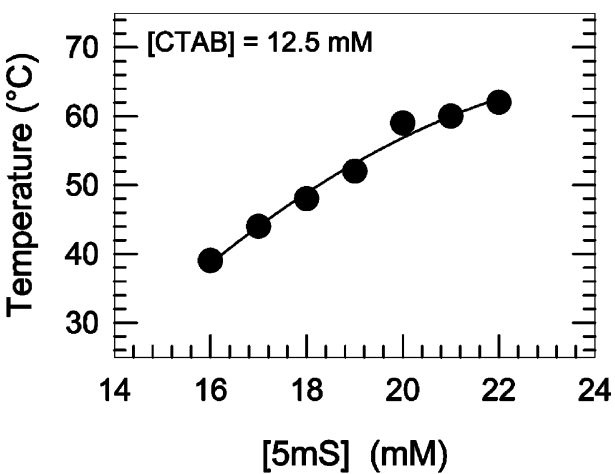

Figure 5. Vesicle-micelle phase transition temperature as a function of $[5 \mathrm{mS}]$ for a fixed concentration of CTAB $(12.5 \mathrm{mM})$.

of the elastic $G^{\prime}$ and viscous $G^{\prime \prime}$ moduli versus frequency $\omega$. The sample again shows a viscoelastic response typical of wormlike micelles - that is, at high frequencies (or short time scales), it behaves elastically $\left(G^{\prime}>G^{\prime \prime}\right)$, while at low frequencies (or long time scales), it switches to a viscous behavior $\left(G^{\prime \prime}\right.$ $>G^{\prime}$, with both moduli varying strongly with frequency). ${ }^{3}$ The longest relaxation time of the sample (inverse of the frequency at which $G^{\prime}$ and $G^{\prime \prime}$ cross) is about $1.4 \mathrm{~s}$ at $55^{\circ} \mathrm{C}$. Figure $4 \mathrm{a}$ also shows that heating the micellar solution from 55 to $60{ }^{\circ} \mathrm{C}$ lowers the zero-shear viscosity by a factor of 2 (the relaxation time in dynamic rheology is lowered by a similar amount; data not shown). Such decreases in viscosity and relaxation time with temperature are expected for wormlike micelles - they arise due to an exponential reduction in the micellar contour length with increasing temperature. ${ }^{2}$

Similar temperature-induced changes in both the phase behavior and rheology were observed for all the vesicle samples investigated. The onset of the vesicle to micelle transition, and thereby the onset of the viscosity increase, could be tuned by varying the solution composition. Figure 5 plots the transition temperature as a function of [5mS] for a fixed [CTAB] of 12.5 $\mathrm{mM}$. These transition temperatures were determined by visual inspection, and they correlate well with the complete disappearance of turbidity in the sample (i.e., with optical density $\rightarrow$ 0 in plots such as Figure 3). We note from Figure 5 that the phase transition systematically shifts to higher temperatures with increasing $[5 \mathrm{mS}]$. It is worth emphasizing also that the transition is thermoreversible, so that vesicles that are disrupted into micelles upon heating can be re-formed upon cooling.

Figure 6 shows the changes in zero-shear viscosity as a function of temperature for three different CTAB $/ 5 \mathrm{mS}$ samples, each with $12.5 \mathrm{mM}[\mathrm{CTAB}]$ and differing $[5 \mathrm{mS}]$. The data again demonstrate that the onset of the transition and, correspondingly, the location of the viscosity peak shift to higher temperatures as $[5 \mathrm{mS}]$ is increased. In addition, the magnitude of the viscosity rise is also seen to be a function of $[5 \mathrm{mS}]$. Thus, the peak viscosity is highest for the $18 \mathrm{mM}$ sample, and the viscosity rise in this case amounts to a factor of about 1500 . The viscosity rise is less appreciable for the $20 \mathrm{mM}$ sample, and an even smaller viscosity increase occurs for the $23 \mathrm{mM}$ one. Similar trends can also be obtained by varying the CTAB concentration in the sample. In sum, both the onset and magnitude of the viscosity increase are tunable via the sample composition.

Changes in solution structure with temperature were also investigated by SANS. Figure 7 shows SANS spectra over a range of temperatures for a sample in $\mathrm{D}_{2} \mathrm{O}$ containing $12.5 \mathrm{mM}$

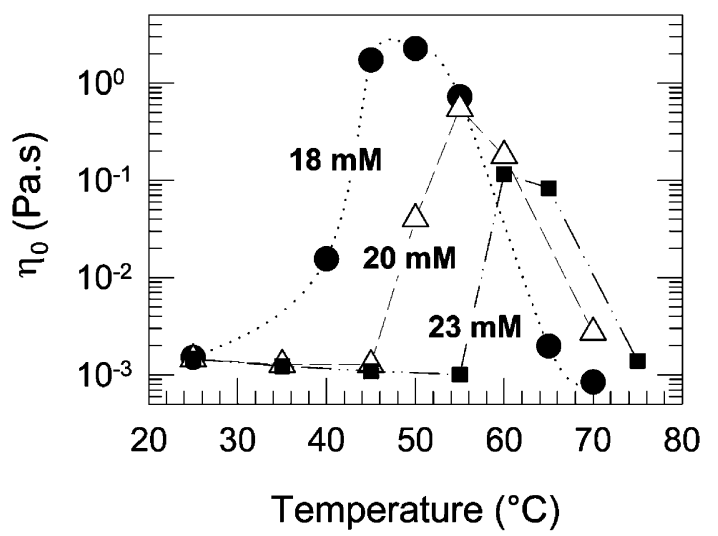

Figure 6. Zero-shear viscosity $\eta_{0}$ as a function of temperature for three CTAB/5mS solutions containing $12.5 \mathrm{mM} \mathrm{CTAB}$ and differing concentrations of $5 \mathrm{mS}$ (indicated along each curve).

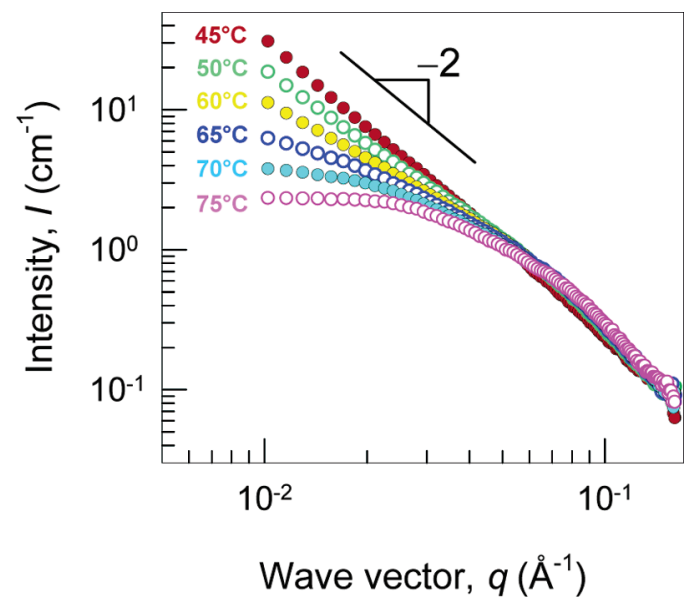

Figure 7. SANS scattering at various temperatures from a $\mathrm{CTAB} / 5 \mathrm{mS}$ solution containing $12.5 \mathrm{mM} \mathrm{CTAB}$ and $20 \mathrm{mM} 5 \mathrm{mS}$. The data indicate the presence of vesicles at temperatures up to $45^{\circ} \mathrm{C}$ (slope of -2 ) and micelles at higher temperatures.

CTAB and $20 \mathrm{mM} 5 \mathrm{mS}$. This $\mathrm{D}_{2} \mathrm{O}$ sample behaves almost identically as its counterpart in $\mathrm{H}_{2} \mathrm{O}$ (Figure 3). SANS spectra for this sample overlap perfectly from 25 to $45^{\circ} \mathrm{C}$, indicating negligible changes in microstructure over this range of temperatures. The data at $45{ }^{\circ} \mathrm{C}$ are plotted in Figure 7 , and the characteristic $q^{-2}$ decay of vesicles is seen. Beyond $45{ }^{\circ} \mathrm{C}$, however, significant changes occur in the spectra. The intensity drops at low $q$, and the $q^{-2}$ decay is no longer observed, indicating a transformation of the vesicular structures. The onset of changes in SANS coincides with the onset of transitions in turbidity and rheology (Figure 3). Thus SANS again supports the occurrence of a vesicle-to-micelle transition with increasing temperature in $\mathrm{CTAB} / 5 \mathrm{mS}$ samples. Further analysis of the SANS data is described below.

\section{Discussion}

SANS Modeling and Analysis. Further information from the SANS data can be obtained by modeling it by the IFT method, described in the experimental section of the Supporting Information. Using this method, the SANS data can be analyzed without assuming a priori if micelles or vesicles are present in the sample. First, we apply the IFT analysis to the SANS data at room temperature $\left(25^{\circ} \mathrm{C}\right)$, presented in Figure 2. The pair distance distribution function, $p(r)$, from IFT is shown for $5 \mathrm{mS}$ concentrations of 10 and $20 \mathrm{mM}$ in Figure 8 . For the $10 \mathrm{mM}$ 


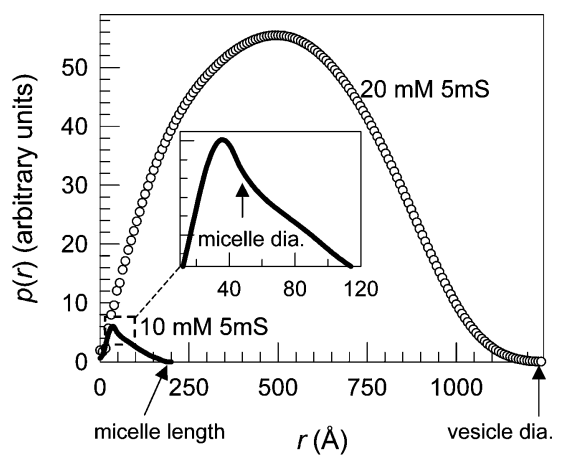

Figure 8. Pair distance distribution functions, $p(r)$, corresponding to the SANS data at room temperature for $\mathrm{CTAB} / 5 \mathrm{mS}$ samples with $12.5 \mathrm{mM}$ $\mathrm{CTAB}$ and two different $5 \mathrm{mS}$ concentrations (original data in Figure 2). The inset is a magnification of the $10 \mathrm{mM}$ data showing the inflection point, followed by linear decrease, in $p(r)$.

sample, $p(r)$ is asymmetrical, with an inflection point around $52 \AA$ (see inset) followed by an approximately linear decrease to zero around $200 \AA$. This $p(r)$ function is characteristic of cylindrical micelles. ${ }^{14,15}$ The inflection point gives the cylinder diameter, while the point where $p(r)$ meets the $x$-axis is an estimate for the average length of the cylinders. Thus, IFT confirms the presence of short, cylindrical (rodlike) micelles in the $10 \mathrm{mM}$ sample.

In contrast, IFT yields a very different $p(r)$ for samples at higher $[5 \mathrm{mS}]$ that fall in the vesicle region of the phase diagram in Figure 1. This is illustrated in Figure 8 for the $20 \mathrm{mM}$ sample, where we see a broad symmetrical peak in $p(r)$. This shape of the $p(r)$ function suggests large symmetrical structures, that is, unilamellar vesicles. ${ }^{14}$ The point where $p(r)$ meets the $x$-axis gives the vesicle diameter, which is $1270 \AA$ in this case. For comparison, measurements by dynamic light scattering (DLS) yield a diameter of $1340 \AA$ for the vesicles in this sample. This is a reasonable agreement, and the data from both techniques point to the existence of vesicular structures in the $20 \mathrm{mM}$ sample with sizes on the order of $130 \mathrm{~nm}$. The vesicle bilayer thickness can be determined independently from the SANS data using a cross-sectional Guinier plot or a cross-sectional IFT analysis (not shown) and is found to be $24.9 \AA$. This value is comparable to the bilayer thicknesses reported for other surfactant vesicles. ${ }^{16}$

Similar IFT analysis was also done on the SANS data presented in Figure 7 for the 12.5/20 CTAB/5mS sample over a range of temperatures. Corresponding plots of $p(r)$ at the various temperatures are shown in Figure 9. In this case, the $p(r)$ is simple to interpret in the limit of low and high temperatures (Figure 9a,c) but is more complicated at the intermediate temperatures (Figure 9b). At low temperatures $\left(25-45^{\circ} \mathrm{C}\right)$, the $p(r)$ is symmetrical and indicative of vesicles. On the other hand, at high temperatures $\left(65-75^{\circ} \mathrm{C}\right)$, the $p(r)$ is asymmetrical and corresponds to cylindrical micelles. Note here that the micelle length (intersections with the $x$-axis, marked by arrows) decreases with temperature, while the micelle diameter (inflection point) remains constant. Finally, turning to the data between 50 and $60{ }^{\circ} \mathrm{C}$, the $p(r)$ plots do not correspond to either vesicles or micelles alone. All three plots show a primary peak and a shoulder, implying a likely

(15) Glatter, O. J. Appl. Crystallogr. 1979, 12, 166-175.

(16) Jung, H. T.; Coldren, B.; Zasadzinski, J. A.; Iampietro, D. J.; Kaler, E. W. Proc. Natl. Acad. Sci. U.S.A. 2001, 98, 1353-1357. coexistence of two distinct structures. Thus, the IFT analysis suggests that over a span of temperatures near the onset of the transition, vesicles coexist with micelles. It is only at higher temperatures that the vesicles are completely transformed into cylindrical micelles.

The above analysis has important implications for the nature of the vesicle-to-micelle phase transition. On the basis of the IFT plots, this transition seems to be a continuous (secondorder) phase transition. Thus, at the onset of the transition, some of the vesicles are transformed into micelles. As temperature is increased, a larger fraction of vesicles undergo this transformation, and this continues until all the vesicles are eventually transformed. The above findings are in broad agreement with earlier studies on vesicle-to-micelle transitions induced by compositional or temperature changes. ${ }^{17-19}$ These studies have found that vesicles and micelles can coexist under certain conditions. Note that there may also be other intermediate morphologies in such cases, such as disklike micelles or bilayers with holes. ${ }^{18-20}$ Direct evidence for the coexistence of disklike micelles with vesicles has been provided by cryo-TEM studies. ${ }^{18}$

Mechanism for Vesicle-Micelle Transition. We now proceed to consider the questions of (a) why vesicles form in $\mathrm{CTAB} / 5 \mathrm{mS}$ mixtures; (b) why the vesicles become micelles upon heating; and (c) what controls the onset of the transition. A possible mechanism is offered here on the basis of the schematics shown in Figure 10. As indicated, the $5 \mathrm{mS}$ molecule will have a strong tendency to bind to the amphiphilic aggregate. ${ }^{8}$ This binding will be stronger than that of the parent salicylic acid because of the presence of the methyl group and its location at the 5-position. Being at the 5-position, the methyl group can become embedded in the hydrophobic part of the aggregate even as the hydrophilic $(\mathrm{OH}$ and $\mathrm{COOH})$ groups at the opposite end of the counterion remain in contact with water. The resultant binding of $5 \mathrm{mS}$ to CTAB will cause a shielding of the headgroup charge and thereby a reduction in effective headgroup area, while the volume of the hydrophobic tail portion will increase. Adding low amounts of $5 \mathrm{mS}$ will thus promote the growth of spherical CTAB micelles into wormlike micelles. ${ }^{8}$ However, when the $5 \mathrm{mS}: \mathrm{CTAB}$ ratio is increased beyond equimolar, the headgroup area could be further reduced and the tail volume increased such that the molecular geometry favors the formation of low-curvature aggregates (i.e., vesicles). Note that, in our experiments, vesicle formation is initiated for 12.5 $\mathrm{mM}$ CTAB only when $[5 \mathrm{mS}]$ is increased above $15 \mathrm{mM}$ (i.e., slightly above equimolar). A similar progression from spherical to rodlike micelles and then to vesicles occurs for mixed cationic/anionic surfactant mixtures as well with increasing molar ratio. ${ }^{13}$

To explain the effect of temperature, we postulate that the binding (adsorption) of $5 \mathrm{mS}$ is a reversible process. ${ }^{10,11}$ At low temperatures, the $5 \mathrm{mS}$ molecules will mostly remain bound to the vesicles. However, upon heating, a fraction of bound $5 \mathrm{mS}$ may tend to desorb from the vesicles, possibly due to changes in the solubility of $5 \mathrm{mS}$ in the bulk water. As mentioned, $5 \mathrm{mS}$ has a limited solubility in water, but this value increases with

(17) Soderman, O.; Herrington, K. L.; Kaler, E. W.; Miller, D. D. Langmuir 1997, 13, 5531-5538.

(18) Xia, Y.; Goldmints, I.; Johnson, P. W.; Hatton, T. A.; Bose, A. Langmuir 2002, 18, 3822-3828.

(19) Leng, J.; Egelhaaf, S. U.; Cates, M. E. Biophys. J. 2003, 85, 1624-1646.

(20) Edwards, K.; Gustafsson, J.; Almgren, M.; Karlsson, G. J. Colloid Interface Sci. 1993, 161, 299-309. 


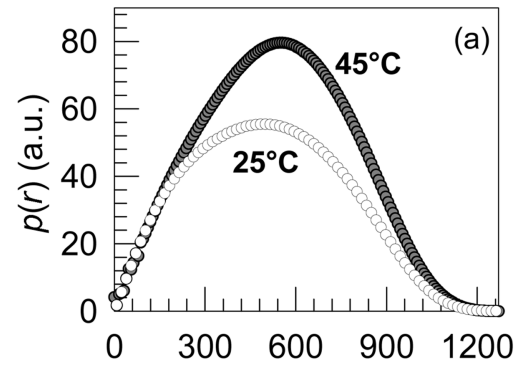

$r(\AA)$
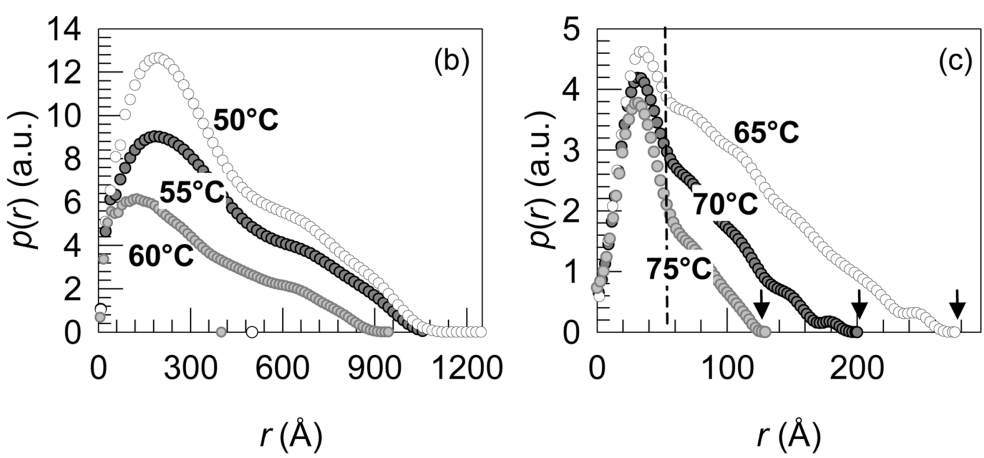

Figure 9. Pair distance distribution functions, $p(r)$, corresponding to the SANS data at various temperatures for a $12.5 \mathrm{mM}$ CTAB $+20 \mathrm{mM} 5 \mathrm{mS}$ sample (original data in Figure 7). As discussed in the text, the $p(r)$ plots indicate the presence of vesicles at low temperatures (a); vesicles + micelles at intermediate temperatures (b); and cylindrical micelles at high temperatures (c).

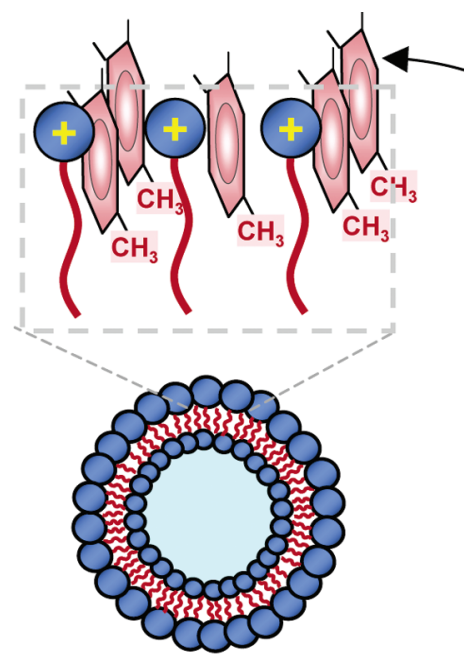

Unilamellar Vesicles
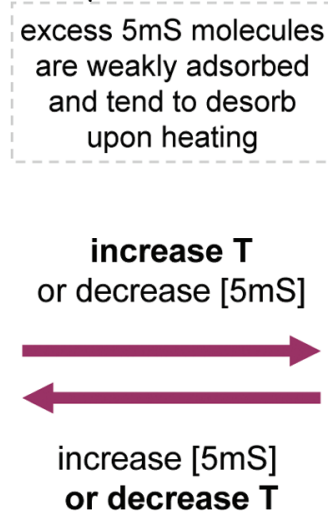

or decrease T

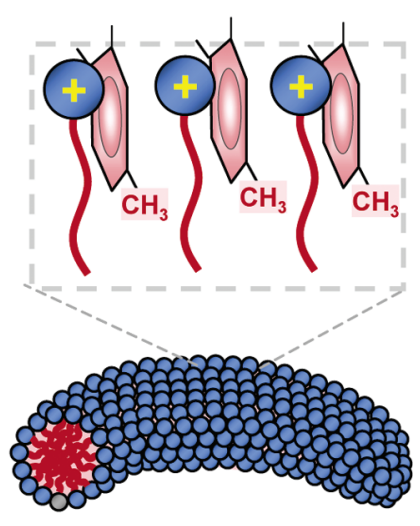

Wormlike Micelles

Figure 10. Mechanism of the vesicle-to-wormlike micelle transition in CTAB/5mS mixtures. Vesicles are formed when there is an excess of $5 \mathrm{mS}$ molecules bound to the aggregate. Some of these molecules are weakly adsorbed at the aggregate interface and tend to desorb upon heating. The desorption-induced change in the molecular geometry of the aggregates induces them to transform into wormlike micelles. (Note: For clarity, the $-\mathrm{OH}$ and $-\mathrm{COOH}$ groups on the $5 \mathrm{mS}$ molecules are not shown.)

temperature. Our measurements (see Supporting Information) show that the solubility of $5 \mathrm{mS}$ in deionized water increases from $7.4 \mathrm{mM}$ at $25^{\circ} \mathrm{C}$ to $18.9 \mathrm{mM}$ at $85^{\circ} \mathrm{C}$. This shows that $5 \mathrm{mS}$ does have an increased affinity for water at higher temperatures, which could help explain its desorption from the vesicles. Such desorption, if it occurs, would increase the effective headgroup area and reduce the tail volume, thereby driving the vesicles to aggregates of higher curvaturespecifically to cylindrical micelles. Such a mechanism could explain the observed results.

The above mechanism allows concentration and temperature effects to be treated in an analogous manner, as shown by Figure 10. Thus, vesicles can be viewed to form when the effective $5 \mathrm{mS}$ concentration at the aggregate interface becomes high. This could be reached either by adding more $5 \mathrm{mS}$ to the solution or by lowering the temperature. Conversely, increasing the temperature would reduce the effective $5 \mathrm{mS}$ concentration at the aggregate interface and thereby induce micelles. Along the same lines, one can also explain the increase in vesicle-micelle transition temperature with increasing $[5 \mathrm{mS}]$ for a constant [CTAB] (Figure 5). This trend could be because, in vesicles with a higher concentration of bound $5 \mathrm{mS}$, more of these bound molecules have to desorb before the transition point can be reached, which requires the sample to be heated to a higher temperature. The above argument suggests that the $[5 \mathrm{mS}]$ : $[\mathrm{CTAB}]$ ratio is the important variable. Indeed, preliminary experiments with different CTAB concentrations (data not shown) confirm that the $[5 \mathrm{mS}]:[\mathrm{CTAB}]$ ratio largely controls the onset of the vesicle-to-micelle transition.

\section{Conclusions}

This study has demonstrated that the aromatic derivative, 5-methyl salicylic acid ( $5 \mathrm{mS})$, can induce the cationic surfactant, $\mathrm{CTAB}$, to form either wormlike micelles or unilamellar vesicles depending on the solution composition. Additionally, CTAB/ $5 \mathrm{mS}$ vesicles can be transformed into long, flexible "wormlike" micelles by heating the solution beyond a critical temperature. The vesicle-to-micelle transition causes the solutions to switch from low-viscosity, Newtonian fluids to viscoelastic, shearthinning fluids. The zero-shear viscosities of these fluids increase dramatically as a result-by more than a factor of 1000 in some cases. SANS data confirm the thermoreversible phase transition from vesicles to micelles, and analysis of the data by the IFT method shows that vesicles coexist with micelles over a range of temperatures close to the transition. A qualitative mechanism to account for the vesicle-to-micelle transition involves the desorption of bound $5 \mathrm{mS}$ molecules from the vesicles as temperature is increased. 
Acknowledgment. This work was funded by a seed grant from the Small Smart Systems Center (SSSC) at UMD and a grant from NSF-CTS. Undergraduate students, David Griffin and Patrick Elder, helped with the experiments, and Dr. Alex Norman provided valuable input on the IFT analysis. We would also like to acknowledge NIST for facilitating the SANS experiments performed as part of this work.
Supporting Information Available: A detailed experimental section for this paper is available. This section also includes a description of the IFT modeling technique used to analyze the SANS data. This material is available free of charge via the Internet at http://pubs.acs.org.

JA060021E 


\title{
Supporting Information for
}

\section{Self-Assembly of Surfactant Vesicles that Transform into Viscoelastic Wormlike Micelles Upon Heating}

\author{
Tanner S. Davies, Aimee M. Ketner and Srinivasa R. Raghavan* \\ Department of Chemical \& Biomolecular Engineering \\ University of Maryland \\ College Park, MD 20742-2111 \\ *Corresponding author. email: sraghava@eng.umd.edu
}

\section{EXPERIMENTAL SECTION}

Materials and Sample Preparation. CTAB was purchased from Sigma-Aldrich (St. Louis, MO), while the 5-methyl salicylic acid (2-hydroxy 5-methyl benzoic acid) was obtained from TCI America (Portland, OR). These chemicals were greater than 98\% in purity and were used as received. Solutions were prepared by adding ultra-pure deionized water from a Millipore water-purification system into weighed quantities of the relevant compounds. The samples were heated to $\sim 65^{\circ} \mathrm{C}$ under continuous stirring for about an hour till the solutions became homogeneous. The solutions were then left to cool overnight while being constantly stirred before any further experimentation. For SANS studies, samples were prepared using the same methods, but with the solvent being $\mathrm{D}_{2} \mathrm{O}$ (99.95\% D, purchased from Cambridge Isotope Laboratories, Andover, MA).

Phase Behavior. Phase behavior as a function of temperature was determined by visual observation of sealed samples placed in a water bath, where the temperature was controlled by a Julabo ${ }^{\mathrm{TM}}$ heater. The transition temperature was recorded as the point at which the sample became completely transparent. 
Turbidimetry. Turbidimetric measurements of the solutions were carried out using a Varian Cary 50 spectrophotometer equipped with a Peltier-controlled thermostated cell holder. Samples were studied in $1 \mathrm{~cm}$ cuvettes and the optical density was measured at a wavelength where neither the CTAB nor the $5 \mathrm{mS}$ had any measurable absorption.

Solubility Studies. The solubility of $5 \mathrm{mS}$ in water at different temperatures was determined as follows. An excess of $5 \mathrm{mS}$ was added to water in a $1 \mathrm{~cm}$ cuvette and the sample was centrifuged for 15 minutes to compact the $5 \mathrm{mS}$ to the bottom of the cuvette. The cuvette was then placed in the thermostated holder of the UV-Vis instrument. The sample was monitored till the absorbance reached a plateau, and this absorbance was converted to a concentration value using the absorbtivity determined from a calibration curve. The same procedure was repeated at different temperatures.

Rheological Studies. Steady and dynamic rheological experiments were performed on an AR2000 stress controlled rheometer (TA Instruments, Newark, DE). Samples were run on a cone-and-plate geometry ( $40-\mathrm{mm}$ diameter, $2^{\circ}$ cone angle) or a couette geometry (rotor of radius $14 \mathrm{~mm}$ and height $42 \mathrm{~mm}$, and cup of radius $15 \mathrm{~mm}$ ). Dynamic frequency spectra were obtained in the linear viscoelastic regime of each sample as determined by dynamic stress-sweep experiments.

Dynamic Light Scattering (DLS). DLS was used to characterize the sizes of vesicles in solution. A Photocor-FC light scattering instrument with a $5 \mathrm{~mW}$ laser source at $633 \mathrm{~nm}$ was used, with the scattering angle being $90^{\circ}$. A logarithmic correlator was used to measure the autocorrelation function, from which the diffusion coefficient was estimated. The apparent hydrodynamic size of the vesicles was obtained from the diffusion coefficient through the Stokes-Einstein relationship. ${ }^{1}$

SANS. SANS measurements were made on the NG-1 $(8 \mathrm{~m})$ beamline at NIST in Gaithersburg, Maryland. Samples were studied at various temperatures in 2-mm quartz cells. Scattering spectra were corrected and placed on an absolute scale using calibration standards provided by NIST. The data is presented as plots of the radially-averaged 
absolute intensity $I$ versus the wave vector $q=(4 \pi / \lambda) \sin (\theta / 2)$, where $\lambda$ is the wavelength of the incident neutrons and $\theta$ is the scattering angle.

SANS Analysis by IFT. SANS data were analyzed by the Indirect Fourier Transform (IFT) method, which requires no a priori assumptions on the nature of the scatterers. ${ }^{2}$ In this method, a Fourier transformation of the scattering intensity $I(q)$ (with incoherent background subtracted) is performed to obtain the pair distance distribution function $p(r)$ in real space. For non-interacting scatterers, the two are related by: ${ }^{2}$

$$
I(q)=4 \pi \int_{0}^{\infty} p(r) \frac{\sin (q r)}{q r} d r
$$

$p(r)$ provides structural information about the scatterers, such as their maximum dimension. IFT analysis was implemented using the commercial PCG software package.

\section{REFERENCES}

[1] Evans, D. F.; Wennerstrom, H. The Colloidal Domain: Where Physics, Chemistry, Biology, and Technology Meet; Wiley-VCH: New York, 2001.

[2] Glatter, O. J. Appl. Crystallogr. 1977, 10, 415-421. 